\title{
Precession of neutrino-cooled accretion disks in gamma-ray burst engines
}

\author{
M. M. Reynoso ${ }^{1, \star}$, G. E. Romero ${ }^{2,3, \star \star}$, and O. A. Sampayo ${ }^{1, \star \star}$ \\ 1 Departamento de Física, Facultad de Ciencias Exactas y Naturales, Universidad Nacional de Mar del Plata, Funes 3350, \\ (7600) Mar del Plata, Argentina \\ e-mail: mreynoso@mdp.edu.ar \\ 2 Instituto Argentino de Radioastronomía, C.C.5, (1894) Villa Elisa, Buenos Aires, Argentina \\ ${ }^{3}$ Facultad de Ciencias Astronómicas y Geofísicas, Universidad Nacional de La Plata, Paseo del Bosque, (1900) La Plata, Argentina
}

Received 21 November 2005 / Accepted 16 March 2006

ABSTRACT

\begin{abstract}
Aims. We study the precession of accretion disks in the context of gamma-ray burst inner engines.
Methods. With an accretion disk model that allows for neutrino cooling, we evaluate the possible periods of disk precession and nutation due to the Lense-Thirring effect.

Results. Assuming jet ejection perpendicular to the disk midplane and a typical intrinsic time dependence for the burst, we find possible gamma-ray light curves with a temporal microstructure similar to what is observed in some subsamples.

Conclusions. We conclude that the precession and nutation of a neutrino-cooled accretion disk in the burst engine might be responsible for some events, especially those with a slow rise and a fast decay.
\end{abstract}

Key words. gamma-ray: bursts - accretion, accretion disks - neutrinos - black hole physics

\section{Introduction}

Currently favored models for gamma-ray burst (GRB) central engines, such as a failed supernova or "collapsar" (Woosley 1993), the merging of compact objects (Paczynski 1986; Eichler et al. 1989; Narayan et al. 1992), and common-envelope evolution in compact binaries (Fryer \& Woosley 1998), lead to the formation of a transient hot and dense accretion disk around a black hole of a few solar masses. Such a disk can be dominantly cooled through neutrino losses. The accretion of matter, with a rate $\sim 0.1-1 M_{\odot} \mathrm{s}^{-1}$, would power the burst, and the energy ejected through relativistic jets is expected to account for the observed GRB light curves. These curves display a wide variety of forms with timescales from milliseconds to minutes. The usual interpretation of this temporal structure is in terms of shocks that convert bulk kinetic energy into internal energy of the particles, which then cool through synchrotron and inverse Compton emission. The shocks can be internal to the jet and due to colliding shells of different Lorentz factors (e.g. Kobayashi et al. 1997; Daigne \& Mochkovitch 1998; Guetta et al. 2001) or the result of interactions with the ambient medium (e.g. Heinz \& Begelman 1999; Fenimore et al. 1996). Among the observed light curves, however, there are some that are hard to explain using the standard model, like those with a slow rise and a fast decay (Romero et al. 1999).

In this paper, we study the possible precession of dense accretion disks, hence of the jets produced in GRBs. We find that for typical sets of parameters, like a black hole with a mass $M_{\mathrm{bh}}=3 M_{\odot}$, a dimensionless spin parameter $a_{*}=0.9$, and accretion rates in the range $\dot{M}=0.1-1 M_{\odot} \mathrm{s}^{-1}$, spin-induced

\footnotetext{
$\star$ Fellow of CONICET, Argentina.

$\star \star$ Member of CONICET, Argentina.
}

precession can occur in the neutrino-cooled disk. The precession of the disk is transmitted to the relativistic jets, which results in the peculiar temporal microstructure of some GRB light curves. In this way we provide a new basis for the precession of GRB jets (see also Blackman et al. 1996; Portegies et al. 1999; Fargion $1999,2004)$ and, in particular, a possible origin of light curves with slower rises than decays.

The structure of this work is as follows. In Sect. 2, we describe the basic equations of a neutrino-cooled accretion disk within the model considered and obtain the disk structure. In Sect. 3, we analyze the precession of the disk due to the LenseThirring effect and how it relates to the disk density and outer radius. In Sect. 4 we focus on the precession of disks cooled by neutrino emission with general properties such as those outlined in Sect. 2. Finally, we present a discussion with examples in Sect. 5 and a summary with our final comments in Sect. 6.

\section{Neutrino-cooled accretion disks}

A neutrino-cooled accretion disk occurs when rotating matter around a compact object (which we take to be a black hole) achieves the transport of its angular momentum outwards, and as it falls in the potential well, it liberates most of its gravitational energy as neutrinos. Central black holes are rapidly rotating in most candidate GRB engine models, and the structure of accretion disks around such compact objects has been extensively studied in different contexts (e.g. Novikov \& Thorne 1973; Riffert \& Herold 1995; Artemova et al. 1996; Belovorodov 1998; Setiawan et al. 2004).

However, to take neutrino losses and transfer into account, we implement a steady-state disk model similar to the one presented by Di Matteo et al. (2002), but with the necessary relativistic correction factors according to Riffert \& Herold (1995), 
due to the presence of a Kerr black hole. As mentioned by Di Matteo et al. (2002) and Popham et al. (1999), although in GRB's engines the accretion rate may vary, it is expected to vary significantly only in the outer disk. Hence it is a good approximation to study the inner neutrino-cooled disk assuming a constant accretion rate in order to obtain its rough properties. We now describe the basic equations on which our accretion model is based. Conservation of mass implies that the accretion rate is

$\dot{M}=-2 \pi r v_{r} \Sigma$,

where $\Sigma=2 \rho H$ is the surface density and $H$ is the disk's half thickness. The angular momentum of the disk material is diminished in magnitude due to the action of viscosity. Thus, conservation of angular momentum in the direction perpendicular to the disk implies

$f_{\phi}(2 \pi r \cdot 2 H) \cdot r=\frac{\Delta L}{\Delta t}$,

where $f_{\phi}$ is the viscous stress and $L$ the disk angular momentum component in the direction perpendicular to the disk midplane. Then, $\Delta L / \Delta t=\dot{M} \Omega r^{2}$, where $\Omega=\sqrt{G M_{\mathrm{bh}} / r^{3}}$ is the Keplerian angular velocity. We adopt the usual prescription for viscosity: $f_{\phi}=-t_{\phi r}=\alpha P$, where $t_{\phi r}$ is the $\phi-r$ component of the viscous stress tensor, $\alpha$ the dimensionless viscosity parameter (Shakura $\&$ Sunyaev 1973), and $P$ the total pressure. The latter includes with the contributions of gas, radiation plus electron-positron pairs, degeneracy, and neutrino pressure, respectively:

$P=\rho \frac{k T}{m_{n}}\left(\frac{1+3 X_{\mathrm{nuc}}}{4}\right)+\frac{11}{12} a T^{4}+K\left(\frac{\rho}{2}\right)^{4 / 3}+\frac{u_{v}}{3}$,

where

$K=\frac{2 \pi h c}{3}\left(\frac{3}{8 \pi m_{n}}\right)^{\frac{4}{3}}$,

$X_{\text {nuc }}$ is the mass fraction of free nucleons specified below, $m_{n}$ is the nucleon mass, $a$ the radiation density constant and $u_{v}$ is the neutrino energy density given by $u_{v}=\sum_{i}\left(\tau_{v_{i}} / 2+1 / \sqrt{3}\right) /\left(\tau_{v_{i}} / 2+\right.$ $\left.1 / \sqrt{3}+1 /\left(3 \tau_{a, v_{i}}\right)\right)$ (Phopam \& Narayan 1995), with $\tau_{i}$ as the neutrino optical depth for the flavor $i$ (see below).

The rate at which energy (entropy) per volume unit is generated by viscosity for a Keplerian disk is $\dot{Q}_{\mathrm{vis}}^{+}=-f_{\phi} t_{\phi r} / v$, where $v$ is the kinematic viscosity coefficient (e.g. Shaphiro \& Teukolsky 1983), so that the heating rate is found to be

$\dot{Q}_{\mathrm{vis}}^{+}=\frac{3 \dot{M} G M_{\mathrm{bh}}}{8 \pi H r^{3}}$.

Solutions for the disk in thermal equilibrium arise when the energy balance is achieved (e.g., Kohri \& Mineshige 2002):

$Q_{\mathrm{vis}}^{+}=Q^{-}$,

where $Q_{\mathrm{vis}}^{+}=\dot{Q}_{\mathrm{vis}}^{+} H$ is the viscous heating rate integrated over the half thickness, and the corresponding cooling term consists in turn of four contributions,

$Q^{-}=Q_{\text {rad }}^{-}+Q_{\text {photo }}^{-}+Q_{\text {adv }}^{-}+Q_{v}^{-}$.

The radiative cooling $Q_{\text {rad }}^{-}$is negligible as compared with the other sources of cooling in the range of high temperatures and densities that we deal with. The photodissociation of heavy nuclei constitutes a cooling mechanism, with a rate approximately given by

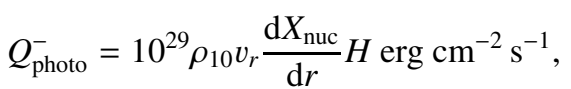

where $\rho_{10}=\rho / 10^{10} \mathrm{~g} \mathrm{~cm}^{-3}$ and

$X_{\text {nuc }} \approx 34.8 \rho_{10}^{-3 / 4} T_{11}^{9 / 8} \exp \left(-0.61 / T_{11}\right)$,

with $T_{11}=T / 10^{11} \mathrm{~K}$ (e.g. Popham et al. 1999).

We approximate the advective cooling term as in Di Mateo et al. (2002; see also Narayan \& Yi 1994)

$Q_{\mathrm{adv}}^{-} \cong v_{r} \frac{H}{r}\left[\frac{38}{9} a T^{4}+\frac{3}{8} \frac{\rho k T}{m_{n}}\left(1+X_{\mathrm{nuc}}\right)\right]$,

where the first term includes both the entropy density of radiation and that of neutrinos.

The neutrino cooling rate integrated over $H$ in the optically thin limit is just

$Q_{v}^{-}=\left(\dot{q}_{\mathrm{e} N}+\dot{q}_{v_{i} \bar{v}_{i}}+\dot{q}_{\text {brem }}+q_{\text {plas }}\right) H$,

where $\dot{q}_{\mathrm{e} N}$ is the cooling rate due to electron or positron capture by a nucleon, $\dot{q}_{v_{i} \bar{v}_{i}}$ is that caused by electron-positron pair annihilation, $\dot{q}_{\text {brem }}$ is the cooling term corresponding to nucleonnucleon bremsstrahlung, and $\dot{q}_{\text {plas }}$ is the rate due to plasmon decays, (Kohri et al. 2005; Di Matteo et al. 2002; Kohri \& Mineshige 2002):

$$
\begin{aligned}
\dot{q}_{N \mathrm{e}} & =9.2 \times 10^{33} \rho_{10} T_{11}^{6} X_{\mathrm{nuc}} \mathrm{erg} \mathrm{cm}^{-3} \mathrm{~s}^{-1} \\
\dot{q}_{v_{\mathrm{e}} \bar{v}_{\mathrm{e}}} & =3.4 \times 10^{33} T_{11}^{9} \mathrm{erg} \mathrm{cm}^{-3} \mathrm{~s}^{-1} \\
\dot{q}_{\nu_{\mu} \bar{v}_{\mu}} & =q_{v_{\tau} \bar{v}_{\tau}}=0.7 \times 10^{33} T_{11}^{9} \mathrm{erg} \mathrm{cm}^{-3} \mathrm{~s}^{-1} \\
\dot{q}_{\text {brem }} & =1.5 \times 10^{27} \rho_{10}^{2} T_{11}^{5.5} \mathrm{erg} \mathrm{cm}^{-3} \mathrm{~s}^{-1} \\
\dot{q}_{\text {plas }} & =3 \times 10^{32} T_{11}^{9} \mathrm{e}^{-\gamma_{\mathrm{p}}}\left(\gamma_{\mathrm{p}}^{6}+\gamma_{\mathrm{p}}^{7}+\frac{\gamma_{\mathrm{p}}^{8}}{2}\right) \mathrm{erg} \mathrm{cm}^{-3} \mathrm{~s}^{-1}
\end{aligned}
$$

In these equations,

$\gamma_{\mathrm{p}}=5.565 \times 10^{-2} \sqrt{\left(\pi^{2}+3 \eta_{\mathrm{e}}^{2}\right) / 3}$

$\eta_{\mathrm{e}}=\mu_{\mathrm{e}} /(k T)$,

and the electron chemical potential $\mu_{e}$ is obtained by solving Eq. (26) of Kohri \& Mineshige (2002).

Without assuming neutrino transparency, it is necessary to consider the inverse processes to Eqs. (12)-(16) that produce absorption of neutrinos, as well as the scattering with nucleons that may prevent the free escape of neutrinos. The absorptive optical depths for the three neutrino flavors are (Kohri et al. 2005)

$$
\begin{aligned}
\tau_{\mathrm{a}, v_{\mathrm{e}}} & =\frac{\left(\dot{q}_{\mathrm{e} N}+\dot{q}_{\mathrm{e}^{-} \mathrm{e}^{+} \rightarrow v_{\mathrm{e}} \overline{\mathrm{e}}_{\mathrm{e}}}+\dot{q}_{\text {brem }}+q_{\mathrm{plas}}\right) H}{(7 / 2) \sigma T^{4}} \\
\tau_{\mathrm{a}, \nu_{\mu}} & =\tau_{\mathrm{a}, \nu_{\tau}}=\frac{\left(\dot{q}_{\mathrm{e}^{-} \mathrm{e}^{+} \rightarrow v_{\mu} \bar{\nu}_{\mu}}+\dot{q}_{\text {brem }}\right) H}{(7 / 2) \sigma T^{4}}
\end{aligned}
$$

whereas for the scattering optical depth, we use the expression given by Di Matteo et al. (2002),

$\tau_{\mathrm{s}, v_{i}} \cong 2.7 \times 10^{-7} T_{11}^{2} \rho_{10} H$.

Then, adopting a simplified model for the neutrino transport (Popham \& Narayan 1995), the neutrino flux integrated over a half thickness is

$Q_{v}^{-}=\sum_{i} \frac{7 / 8 \sigma T^{4}}{(3 / 4)\left(\tau_{i} / 2+1 / \sqrt{3}+1 /\left(3 \tau_{\mathrm{a}, i}\right)\right)}$

where $\tau_{i}=\tau_{\mathrm{a}, i}+\tau_{\mathrm{s}, i}$. We employ this last expression with which the neutrino emission is correctly obtained in situations with both small and large optical depths. 
For the purposes of this paper, we need to work out the structure of a steady-state accretion disk around a rotating black hole with a mass $M_{\mathrm{bh}}=3 M_{\odot}$, a dimensionless spin parameter $a_{*}=0.9\left(a_{*}=1\right.$ would be a maximally rotating black hole), a viscosity parameter $\alpha=0.1$, and an accretion rate between $\dot{M}=0.1-1 M_{\odot} \mathrm{s}^{-1}$. It is necessary to introduce general relativistic correction factors to some of the equations presented above. These factors are (Riffert \& Herold 1995):

$$
\begin{aligned}
& A=1-\frac{2 G M_{\mathrm{bh}}}{c^{2} r}+\left(\frac{G M_{\mathrm{bh}} a_{*}}{c^{2} r}\right)^{2} \\
& B=1-\frac{3 G M_{\mathrm{bh}}}{c^{2} r}+2 a_{*}\left(\frac{G M_{\mathrm{bh}} a_{*}}{c^{2} r}\right)^{3 / 2} \\
& C=1-4 a_{*}\left(\frac{G M_{\mathrm{bh}} a_{*}}{c^{2} r}\right)^{3 / 2}+3\left(\frac{G M_{\mathrm{bh}} a_{*}}{c^{2} r}\right)^{2} \\
& D=\int_{r_{\mathrm{ms}}}^{r} \frac{\frac{x^{2} c^{4}}{8 G^{2}}-\frac{3 x M_{\mathrm{bh}} c^{2}}{4 G}+\sqrt{\frac{a_{*}^{2} M^{3} c^{2} x}{G}}-\frac{3 a_{*}^{2} M_{\mathrm{bh}}^{2}}{8}}{\frac{\sqrt{r x}}{4}\left(\frac{x^{2} c^{4}}{G^{2}}-\frac{3 x M_{\mathrm{bh}} c^{2}}{G}+2 \sqrt{\frac{a_{*}^{2} M^{3} c^{2} x}{G}}\right)} \mathrm{d} x .
\end{aligned}
$$

Our Eq. (1) remains valid, while hydrostatic equilibrium in the vertical direction leads to a corrected expression for the half thickness of the disk (Riffert \& Herold 1995):

$H \simeq \sqrt{\frac{P r^{3}}{\rho G M_{\mathrm{bh}}}} \sqrt{\frac{B}{C}}$.

The viscous shear $f_{\phi}$ of Eq. (2) is also corrected by

$f_{\phi}=\alpha P \sqrt{\frac{A}{B C}}=\frac{\dot{M}}{4 \pi H} \sqrt{\frac{G M_{\mathrm{bh}}}{r^{3}}} \frac{D}{A}$,

and the energy balance is affected through the correction in the heating rate,

$\dot{Q}_{\mathrm{vis}}^{+}=\frac{3 \dot{M} G M_{\mathrm{bh}}}{8 \pi H r^{3}} \frac{D}{B}$.

With a fixed radius, we numerically solve for $H$ and $P$ Eqs. (27) and (28), which depend only on $\rho$, and using the equation of state (3) we get $T(\rho)$. Then, when the equality (6) is satisfied, the corresponding values of $T$ and $\rho$ are selected for that radius. In Fig. 1 we show the temperature, density, and half thickness profiles for accretion rates of $0.1 M_{\odot} \mathrm{s}^{-1}$ and $1 M_{\odot} \mathrm{s}^{-1}$, and in Fig. 2 we present the neutrino cooling parameter $f_{v} \equiv Q_{v}^{-} / Q_{\mathrm{vis}}^{+}$ and the advection parameter $f_{v} \equiv Q_{\text {adv }}^{-} / Q_{\mathrm{vis}}^{+}$. We observe that for a wide range of disk radii the most important cooling mechanism is neutrino emission with advection in the second place, dominating the latter only in the first $\sim 2 \mathrm{~km}$ from the inner radius $\left(R_{\mathrm{ms}} \simeq 10.6 \mathrm{~km}\right)$ as a consequence of the relativistic corrections made. Elsewhere, up to $\sim 200-750 \mathrm{~km}$ depending on the accretion rate, neutrino cooling dominates.

\section{Spin-induced precession of accretion disks}

A Kerr black hole has been found to produce the Lense-Thirring effect (Lense \& Thirring 1918), or dragging of inertial frames, which induces the precession of a particle orbit that happens to be inclined with respect to the black hole equator. The angular momentum of a Kerr black hole of mass $M_{\mathrm{bh}}$ is $J=G M_{\mathrm{bh}}\left|a_{*}\right| / c$, where $a_{*}$ is the dimensionless spin parameter. In such a case, an inclined particle orbit has been found to precess with a frequency $w_{\mathrm{LT}}=G J /\left(c^{2} r^{3}\right)$ (Wilkins 1972).

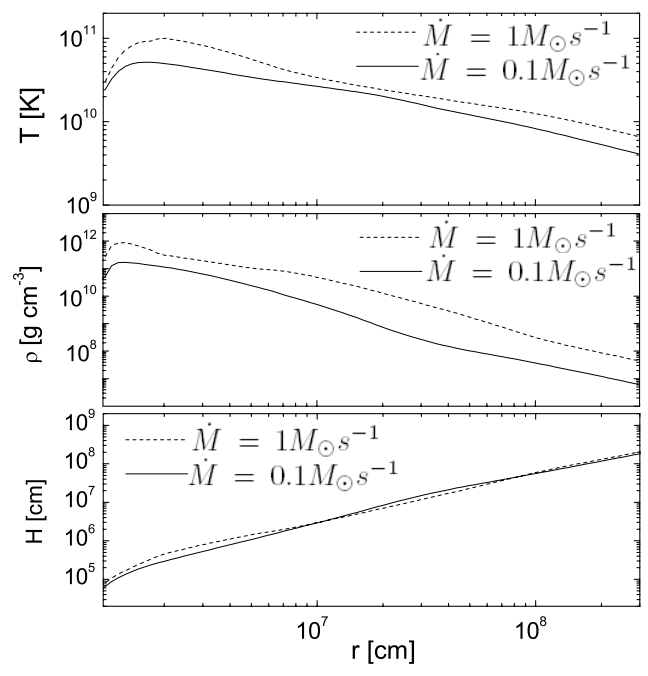

Fig. 1. Disk temperature profile in the upper panel, density profile in the middle panel, and half thickness in the lower panel. Dashed line: $\dot{M}=1 M_{\odot} \mathrm{s}^{-1}$, solid line: $\dot{M}=0.1 M_{\odot} \mathrm{s}^{-1}$.

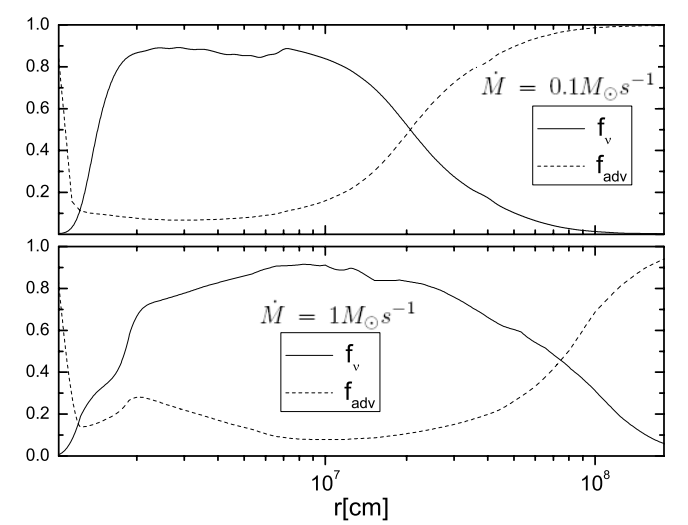

Fig. 2. Neutrino cooling parameter $f_{v}$ (solid line) and advection parameter $f_{\text {adv }}$ (dashed line) in the upper panel for $\dot{M}=0.1 M_{\odot} \mathrm{s}^{-1}$ and in the lower panel for $\dot{M}=1 M_{\odot} \mathrm{s}^{-1}$.

For the candidate central engines of GRB, namely collapsars, the common envelope evolution of a black hole that causes the tidal disruption of a companion helium core, or other types of merging events, the formation of an accretion disk inclined with respect to the Kerr black hole equator cannot be ruled out. In the last two cases, it is clear that the black hole angular momentum $\boldsymbol{J}$ does not need to be aligned with the one of its companion, and in the case of collapsars, when the core collapses to form a black hole, it could happen that the rest of the rapidly rotating star may not be symmetrically distributed in the presence of magnetic fields, leading to the formation of an accretion disk whose angular momentum $\boldsymbol{L}$ would not be exactly aligned with that of the black hole.

In any of the above situations, the inclined disk is also expected to undergo the Bardeen-Patterson effect (Bardeen \& Patterson 1975), that results from the action of viscous torques together with the Lense-Thirring effect. This causes an alignment of the inner parts of the disk with the black hole equator, which arises up to a certain transition radius that depends on the midplane Mach number of the disk (Nelson \& Papaloizou 2000). In the case considered here, the midplane Mach number is $\mathcal{M}<5$, which corresponds to essentially no warping of the disk according to Nelson \& Papaloizou (2000). Also, the disk is expected to precess as a solid body if the sound crossing 


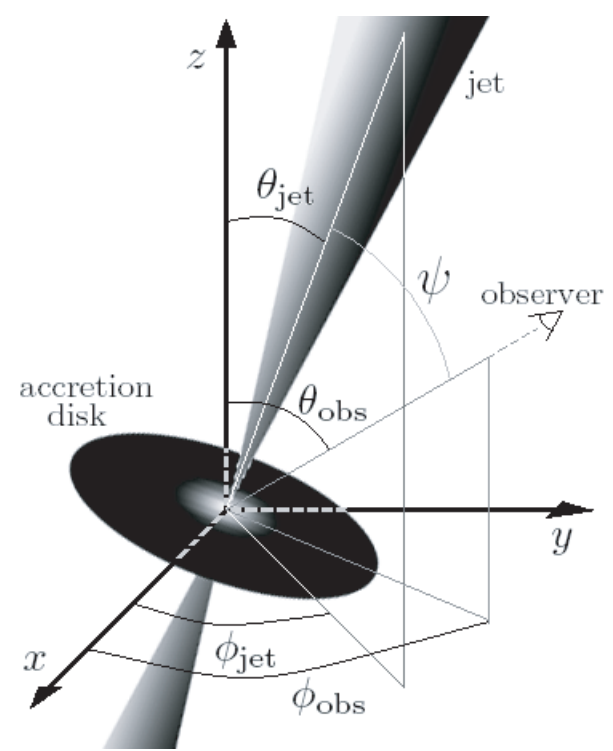

Fig. 3. Schematic picture of the precessing disk and jet. The observer is at infinity in the direction signaled.

timescale (here $\tau_{\text {cross }}<3.2 \times 10^{-2} \mathrm{~s}$ ) is shorter than the precession period (Papaloizou \& Terquem 1995). For precession periods $\tau_{\mathrm{p}}>\tau_{\text {cross }}$, we will then assume rigid body precession, and since there is a torque causing the precession, considering precession alone is actually an approximation, meaning that the same torque should also cause a nutation motion to develop (Goldstein et al. 2002).

Assuming then that the disk supports a strong magnetic field that threads the central black hole, rotational energy can be extracted from the black hole due to mechanisms such as those proposed by Blandford \& Znajek (1977) or by Blandford \& Payne (1982). As the magnetic field is expected to be anchored in the disk, this would lead to the formation of jets perpendicular to the midplane of the disk, so that the precession and nutation of the disk automatically implies the precession and nutation of jets. Then, to kinematically describe the jet motion, we consider the angular evolution of its spherical angles $\theta_{\text {jet }}(t)$ and $\phi_{\text {jet }}(t)$ as in Portegies et al. (1999),

$\phi_{\text {jet }}(t)=\phi_{\text {jet }}(0)+\Omega_{\mathrm{p}} t+\frac{\Omega_{\mathrm{p}}}{\Omega_{\mathrm{n}}} \sin \left(\Omega_{\mathrm{n}} t\right)$,

$\theta_{\text {jet }}(t)=\theta_{\text {jet }}^{0}+\frac{\Omega_{\mathrm{p}}}{\Omega_{\mathrm{n}}} \tan \theta_{\text {jet }}^{0} \cos \left(\Omega_{\mathrm{n}} t\right)$,

where $\Omega_{\mathrm{p}}=2 \pi / \tau_{\mathrm{p}}$, and $\Omega_{\mathrm{n}}=2 \pi / \tau_{\mathrm{n}}$ are the precession and nutation angular frequencies with periods $\tau_{\mathrm{p}}$ and $\tau_{\mathrm{n}}$, respectively. A sketch of the situation is shown in Fig. 3 .

Considering the above expressions (30), we intend to relate the possible precession and nutation periods to some parameter of a given disk, e.g. the surface density $\Sigma(r)$. We do this in a similar way to Caproni et al. (2004), but here nutation will also be considered along with precession. Without nutation, the precession period $\tau_{\mathrm{p}}$ can be estimated to be

$\tau_{\mathrm{p}}=\int_{0}^{2 \pi} \frac{L}{T} \sin \theta \mathrm{d} \phi=2 \pi \sin \theta \frac{L}{T}$

where the magnitudes of the disk angular momentum $L$ and of the precessional torque $T$ applied to the disk are

$L=2 \pi \int_{R_{\mathrm{ms}}}^{R_{\mathrm{out}}} \Sigma(r) \Omega_{\mathrm{k}}(r) r^{3} \mathrm{~d} r$
$T=4 \pi^{2} \sin \theta \int_{R_{\mathrm{ms}}}^{R_{\mathrm{out}}} \Sigma(r) \Omega_{\mathrm{k}}(r) v_{\mathrm{p}, \theta}(r) r^{3} \mathrm{~d} r$.

Here,

$\Omega_{\mathrm{k}}(r)=\frac{c^{3}}{G M_{\mathrm{bh}}}\left[\left(\frac{r}{R_{\mathrm{g}}}\right)^{3 / 2}+a_{*}\right]^{-1}$

is the relativistic Keplerian angular velocity, $R_{\mathrm{g}}=G M_{\mathrm{bh}} / c^{2}$ the gravitational radius, and

$v_{\mathrm{p}, \theta}=\frac{\Omega_{\mathrm{k}}(R)}{2 \pi}\left[1-\sqrt{1 \mp 4 \sqrt{\frac{R_{\mathrm{g}} a_{*}^{2}}{r}}+3\left(\frac{R_{\mathrm{g}} a_{*}}{r}\right)^{2}}\right]$

the nodal frequency that comes from perturbing a circular orbit in the Kerr metric (Kato 1990). The precessing part of the disk ends at an outer radius $R_{\text {out }}$, extending from an inner radius $R_{\mathrm{ms}}=\xi_{\mathrm{ms}} R_{\mathrm{g}}$, where

$\xi_{\mathrm{ms}}=3+A_{2} \mp\left[\left(3-A_{1}\right)\left(3+A_{1}+2 A_{2}\right)\right]^{1 / 2}$,

with

$A_{1}=1+\left(1-a_{*}^{2}\right)^{1 / 3}\left[\left(1+a_{*}\right)^{1 / 3}+\left(1-a_{*}\right)^{1 / 3}\right]$,

and

$A_{2}=\left(3 a_{*}^{2}+A_{1}^{2}\right)^{1 / 2}$,

where the minus sign in $\xi_{\mathrm{ms}}$ corresponds to prograde motion $\left(a_{*}>0\right)$, whereas the plus sign corresponds to retrograde motion $\left(a_{*}<0\right)$. Note that for $a_{*}=0.9, R_{\mathrm{ms}} \simeq 1.1 \times 10^{6} \mathrm{~cm}$.

With both nutation and precession, according to (30),

$\phi\left(\tau_{\mathrm{p}}\right)=2 \pi+\left(\tau_{\mathrm{n}} / \tau_{\mathrm{p}}\right) \sin \left(2 \pi \tau_{\mathrm{p}} / \tau_{\mathrm{n}}\right)$

so we estimate

$$
\begin{aligned}
\tau_{\mathrm{p}}= & \int_{0}^{\phi\left(\tau_{\mathrm{p}}\right)} \frac{L}{T} \sin \theta(t) \mathrm{d} \phi \\
\tau_{\mathrm{p}}= & \frac{2 \pi G M_{\mathrm{bh}}}{c^{3}}\left[2 \pi+\frac{\sin \left(2 \pi R_{\Omega}\right)}{R_{\Omega}}\right] \\
& \times \frac{\int_{\xi_{\mathrm{ms}}}^{\xi_{\text {out }}} \Sigma(\xi)[\Gamma(\xi)]^{-1} \xi^{3} \mathrm{~d} \xi}{\int_{\xi_{\mathrm{ms}}}^{\xi_{\mathrm{mut}}} \Sigma(\xi) \Psi(\xi)[\Gamma(\xi)]^{-2} \xi^{3} \mathrm{~d} \xi}
\end{aligned}
$$

where $R_{\Omega}=\frac{\tau_{\mathrm{p}}}{\tau_{\mathrm{n}}}, \xi=r / R_{\mathrm{g}}, \Gamma(\xi)=\xi^{1 / 2}+a_{*}$, and

$\Psi(\xi)=1-\left(1 \mp 4 a_{*} \xi^{-3 / 2}+3 a_{*}^{2} \xi^{-2}\right)^{1 / 2}$.

\section{Results}

We intend to evaluate the possible precession and nutation periods that are consistent with the typical parameters of an accretion disk which is to power a GRB, i.e. $M_{\mathrm{bh}}=3 M_{\odot}$, $\dot{M}=0.1-1 M_{\odot} \mathrm{s}^{-1}, \alpha=0.1$, and $a_{*} \sim 0.9$. Then, taking into account a disk surface density obtained as in Sect. 2 by $\Sigma(r)=2 \rho(r) H(r)$, we compute the possible precession periods using (41) with $a_{*}=0.9$ for a wide range in $R_{\text {out }}\left(\sim 10^{6}-10^{9} \mathrm{~cm}\right)$ and also for the relative fraction $R_{\Omega}=\tau_{\mathrm{p}} / \tau_{\mathrm{n}}$ varying between $10^{-3}$ and 10 . We thus obtain the precession period $\tau_{\mathrm{p}}$ as a function of both the outer radius $R_{\text {out }}$ of the accretion disk and the fraction $R_{\Omega}$. In Fig. 4 we show the results for an accretion rate of $\dot{M}=0.1 M_{\odot} \mathrm{s}^{-1}$. 


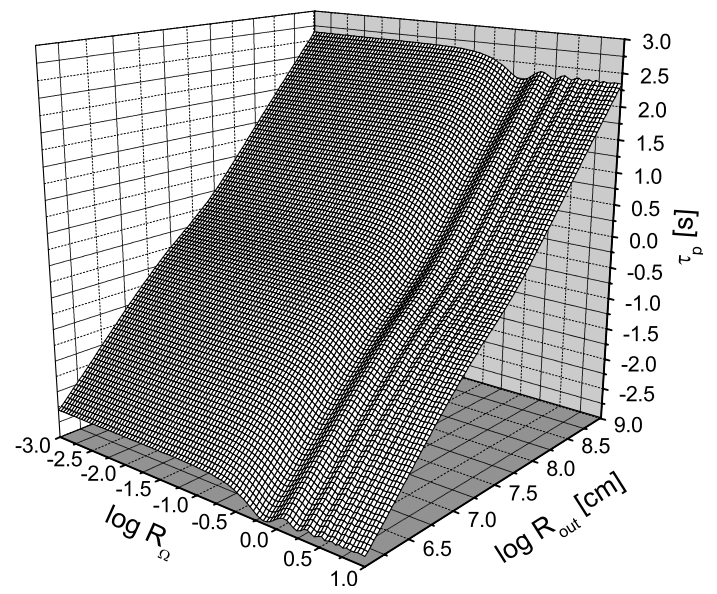

Fig. 4. Precession period vs. $R_{\text {out }}$ and $R_{\Omega}=\tau_{\mathrm{n}} / \tau_{\mathrm{p}}$ for $\dot{M}=0.1 M_{\odot} \mathrm{s}^{-1}$.

Mac Fayden \& Woosley (1999) note that a steady disk may form within the collapsar model at $r \sim 2-3 \times 10^{7} \mathrm{~cm}$, so looking at $R_{\text {out }}$ of that order may give a rough idea of the possible precession periods. Then, by fixing $R_{\Omega}$ at $10,0.1$ and 0.01 , we find the precession period $\tau_{\mathrm{p}}$ as a function of the outer radius $R_{\text {out }}$ for nutation periods of $\tau_{\mathrm{n}}=\tau_{\mathrm{p}} / 10, \tau_{\mathrm{p}} / 0.1$, and $\tau_{\mathrm{p}} / 0.01$, respectively. The results are shown in Fig. 5 for $\dot{M}=0.1 M_{\odot} \mathrm{s}^{-1}$ and $\dot{M}=1 M_{\odot} \mathrm{s}^{-1}$.

It has been found that neutrino emission is important in the inner regions of the disk where very high temperatures and densities are achieved (Di Matteo 2002; Kohri \& Mineshige 2002), in agreement with our results. In particular, from Fig. 2, we observe that neutrino emission dominates until a radius of $\sim 2 \times 10^{7} \mathrm{~cm}$ for $\dot{M}=0.1 M_{\odot} \mathrm{s}^{-1}$ and up to $7.5 \times 10^{7} \mathrm{~cm}$ for $\dot{M}=1 M_{\odot} \mathrm{s}^{-1}$, so these neutrino-cooled accretion disks may precess approximately with periods (see Fig. 5)

$$
\begin{aligned}
& \left.\tau_{\mathrm{p}}\right|_{0.1 M_{\odot} \mathrm{s}^{-1}}<0.38 \mathrm{~s} \text { for } R_{\Omega} \simeq 0.01, \\
& \left.\tau_{\mathrm{p}}\right|_{0.1 M_{\odot} \mathrm{s}^{-1}}<0.41 \mathrm{~s} \text { for } R_{\Omega} \simeq 0.1, \\
& \left.\tau_{\mathrm{p}}\right|_{0.1 M_{\odot} \mathrm{s}^{-1}}<0.2 \mathrm{~s} \text { for } R_{\Omega} \simeq 10,
\end{aligned}
$$

and

$$
\begin{aligned}
& \left.\tau_{\mathrm{p}}\right|_{1 M_{\odot} \mathrm{s}^{-1}}<6.21 \mathrm{~s} \text { for } R_{\Omega} \simeq 0.01, \\
& \left.\tau_{\mathrm{p}}\right|_{1 M_{\odot} \mathrm{s}^{-1}}<6.04 \mathrm{~s} \text { for } R_{\Omega} \simeq 0.1, \\
& \left.\tau_{\mathrm{p}}\right|_{1 M_{\odot} \mathrm{s}^{-1}}<3.12 \mathrm{~s} \text { for } R_{\Omega} \simeq 10 .
\end{aligned}
$$

We note that, for example, if $R_{\Omega}=0.1$, intermediate precession periods of $0.41 \mathrm{~s}$ and $6.04 \mathrm{~s}$ can also be achieved with intermediate values of $\dot{M}$ between $0.1 M_{\odot} \mathrm{s}^{-1}$ and $1 M_{\odot} \mathrm{s}^{-1}$ for different outer radii between $2 \times 10^{7} \mathrm{~cm}$ and $7.5 \times 10^{7} \mathrm{~cm}$.

Now, to work out possible GRB light curves consistent with such precessing neutrino-cooled disks, we proceed as do Portegies et al. (1999) and assume the production of jets perpendicular to the plane of the disk. The spin angular momentum of the black hole $\boldsymbol{J}$ is along the $z$-axis, we fix the observer at the angles $\left(\theta_{\text {obs }}, \phi_{\text {obs }}\right)$, and then let the jet angles $\left(\theta_{\text {jet }}(t), \phi_{\text {jet }}(t)\right)$ evolve according to (30). The angle between the observer and the jet is $\psi=\cos ^{-1}\left(\hat{r}_{\text {obs }} \cdot \hat{r}_{\text {jet }}\right)$ (see Fig. 3), and, following Portegies et al. (1999), we implement the distribution of the corresponding Poynting flux $L(\psi)$ normalized to unity at its maximum (Fig. 6, upper panel). Also, we assume an intrinsic time dependence for the emission $I(t)$, normalized to unity at its maximum as well, which is inspired by what is expected from a typical explosive
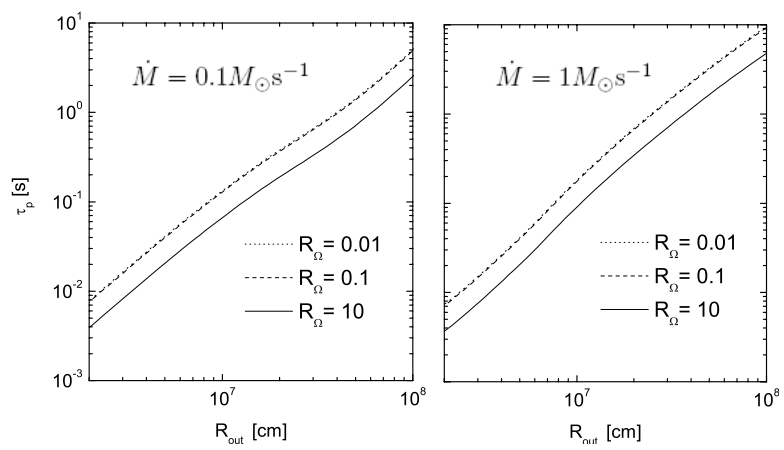

Fig. 5. Precession period as a function of the disk outer radius for $R_{\Omega}=$ $\{0.01,0.1,10\}$. Left panel shows the results for $\dot{M}=0.1 M_{\odot} \mathrm{s}^{-1}$, and the right panel for $\dot{M}=1 M_{\odot} \mathrm{s}^{-1}$.
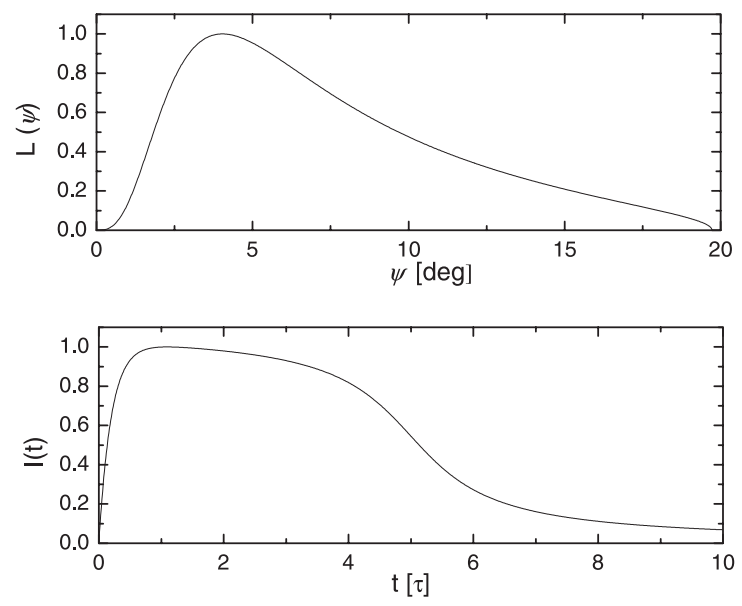

Fig. 6. Upper panel: jet luminosity $L$ as a function of the angle $\psi$ between the jet and the observer. Lower panel: intrinsic time dependence of the burst.

injection, and consists of a fast rise, a plateau, and an exponential decay (Fig. 6, lower panel). The intrinsic duration there was considered to be $10 \mathrm{~s}$, but it could change from burst to burst. Defining $F(t) \equiv I(t) L(\psi)$, we show in Fig. 7 some possible light curves varying the initial jet angles and the observer angles, for different values of $\tau_{\mathrm{p}}\left(R_{\mathrm{out}}, \tau_{\mathrm{n}}\right)$ and for relatively high accretion rates. More signals are plotted in Fig. 8 for lower accretion rates. All these curves have been averaged every $64 \mathrm{~ms}$ to simulate the resolution of the detector.

\section{Discussion}

Inspecting the light curves shown in both Figs. 7 and 8, and many others that can be generated in a similar way, we see that some peculiar time profiles can be produced. For instance, a fast-rise and exponential-decay (FRED) type of burst, with superposed microstructure with timescales of $\delta t \sim 0.1 \mathrm{~s}$, as it has been observed in several cases, can be seen in Fig. 8, upper panel. One interesting type of burst that can not be accommodated in the usual internal shock models is formed for those socalled peculiar asymmetric bursts (PABs). These bursts represent around $\sim 4 \%$ of the total sample (Romero et al. 1999) and present slower risings than decays. In Fig. 7, bottom panel, and Fig. 8, 3rd panel, we show examples of bursts with such a time profile that can result from precessing jets with long nutation periods. In such systems the jet crosses the line of sight before disappearing quite quickly in the observer's frame. For the right 


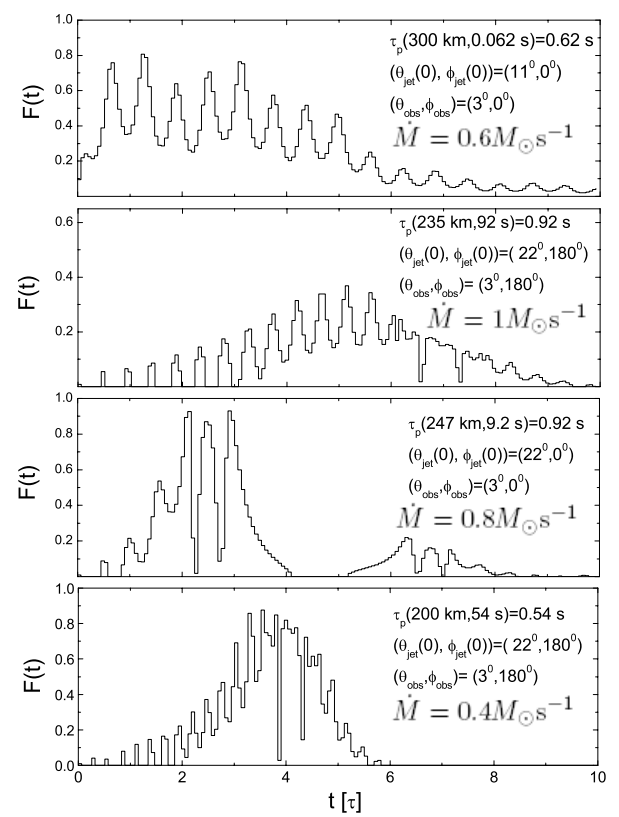

Fig. 7. Some possible GRB light curves for precessing neutrinocooled disks with $0.6 M_{\odot} \mathrm{s}^{-1}$ (upper panel), $1 M_{\odot} \mathrm{s}^{-1}$ (second panel), $0.8 M_{\odot} \mathrm{s}^{-1}$ (third panel) and $0.4 M_{\odot} \mathrm{s}^{-1}$ (bottom panel). The precession period is indicated as $\tau_{\mathrm{p}}\left(R_{\text {out }}, \tau_{\mathrm{n}}\right)$, the jet angles at $t=0$ as $\left(\theta_{\text {jet }}(0), \phi_{\text {jet }}(0)\right)$ and the observer angles as $\left(\theta_{\text {obs }}, \phi_{\text {obs }}\right)$.

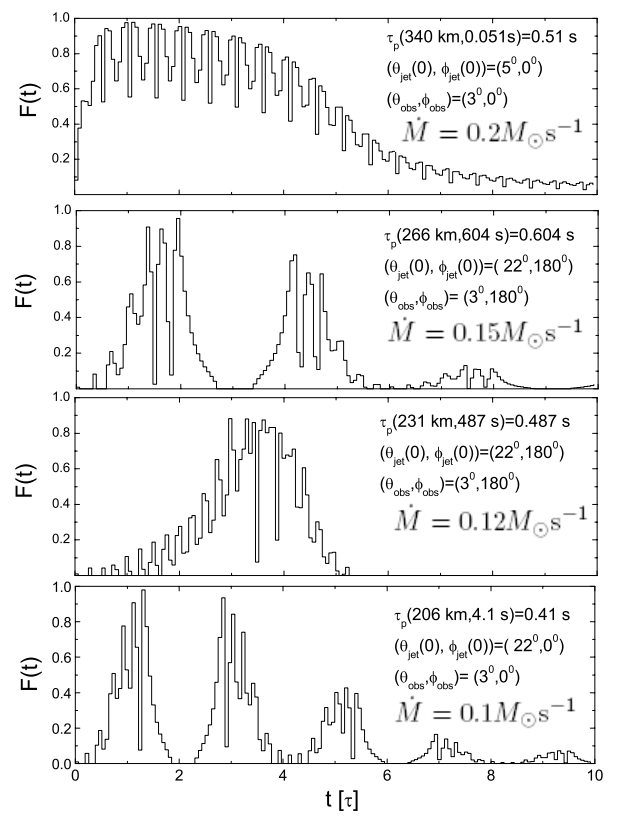

Fig. 8. Some possible GRB light curves for precessing neutrino-cooled disks with $0.2 M_{\odot} \mathrm{s}^{-1}$ (upper panel), $0.15 M_{\odot} \mathrm{s}^{-1}$ (second panel), $0.12 M_{\odot} \mathrm{s}^{-1}$ (third panel) and $0.1 M_{\odot} \mathrm{s}^{-1}$ (bottom panel). The precession period is indicated as $\tau_{\mathrm{p}}\left(R_{\mathrm{out}}, \tau_{\mathrm{n}}\right)$, the jet angles at $t=0$ as $\left(\theta_{\text {jet }}(0), \phi_{\text {jet }}(0)\right)$ and the observer angles as $\left(\theta_{\text {obs }}, \phi_{\text {obs }}\right)$.

combination of viewing angle, precession, and nutation, this results into a typical PAB.

Distinguishing the effects of precession from those of multiple shocks could be more difficult in more conventional light curves with many peaks. However, an analysis of the spectral variability might help at this point, since changes due to precession are essentially geometric and, hence, achromatic. Inverting the problem, clear identification of precession in a given time profile of a GRB can be used to infer some characteristics of the accretion disk, such as its outer radius.

\section{Summary}

We have studied the precession of accretion disks with neutrino losses, taking into account our results obtained for the disk structure by adequately correcting the governing equations of mass conservation, energy balance, hydrostatic equilibrium in the vertical direction, and angular momentum conservation in the presence of a Kerr black hole. Assuming that precession will not significantly alter the obtained disk structure and that it will continue to be determined mainly by the neutrino cooling processes, our results imply that precession and nutation of such disks is possible in the context of GRB engines, giving rise to a temporal microstructure that is similar to what is observed in some cases. In particular, a peculiar behavior that has been observed consisting of a slow rise and a fast decay (Romero et al. 1999) can be obtained in situations with long nutation periods where the jet crosses the line of sight quite suddenly and never reaches it again.

Acknowledgements. This work was supported by the Argentinian agencies CONICET (PIP 5375/05 and PIP 477/98), and ANPCyT (PICT 03-13291 and PICT02 03-11311)

\section{References}

Artemova, I. V., Björnsson, G., \& Novikov, I. D. 1996, ApJ, 461, 565

Bardeen, J. M., \& Patterson, J. A. 1975, ApJ, 195, L65

Belovorodov, A. M. 1998, MNRAS, 297, 739

Blackman, E. G., Yi, I., \& Field, G. B. 1996, ApJ, 473, L79

Blandford, R. D., \& Payne, D. G. 1982, MNRAS, 199, 883

Blandford, R. D., \& Znajek, R. L. 1977, MNRAS, 179, 433

Caproni, A., Mosquera Cuesta, H. J., \& Abraham, Z. 2004, ApJ, 616, L99

Daigne, F., \& Mochkovitch, R. 1998, MNRAS, 296, 275

Di Matteo, T., Perna, R., \& Narayan, R. 2002, ApJ, 579, 706

Eichler, D., Livio, M., Piran, T., \& Schramm, D. N. 1989, Nature, 340, 126

Fargion, D. 1999, in 26th ICRC, OG2.3.14, ed. D. Kieda, M. Salamon, \& B. Dingus, 32 [arXiv: astro-ph/9906432]

Fargion, D. 2004, ASP Conf. Ser., 312, 423

Fenimore, F. E., Madras, C. D., \& Nayakshin, S. 1996, ApJ, 473, 998

Fryer, C. L., \& Woosley, S. E. 1998, ApJ, 502, L9

Goldstein, H., Poole, C. P., \& Safko, J. L. 2002, Classical Mechanics (Massachusetts: Addison-Wesley), 218

Guetta, D., Spada, M., \& Waxman, E. 2001, ApJ, 557, 399

Heinz, S., \& Begelman, M. C. 1999, ApJ, 527, L35

Kato, S. 1990, PASJ, 42, 99

Kobayashi, S., Piran, T., \& Sari, R. 1997, ApJ, 490, 92

Kohri, K., \& Mineshige, S. 2002, ApJ, 577, 311

Kohri, K., Narayan, R., \& Piran, T. 2005, ApJ, 629, 341

Lense, J., \& Thirring, H. 1918, Phys. Z., 19, 156

MacFayden, A. I., \& Woosley, S. E. 1999, ApJ, 524, 262

Narayan, R., \& Yi, I. 1994, ApJ, 428, L13

Narayan, R., Paczynski, B., \& Piran, T. 1992, ApJ, 395, L83

Nelson, R. P., \& Papaloizou, J. C. B. 2000, MNRAS, 315, 570

Novikov, I. D., \& Thorne, K. S. 1973, in Black Holes, Les Astres Occlus, ed. B. \& C. DeWitt (New York: Gordon \& Breach), 343(NT73)

Paczynski, B. 1986, ApJ, 308, L43

Papaloizou, J. C. B., \& Terquem, C. 1995, MNRAS, 274, 987

Popham, R., \& Narayan, R. 1995, ApJ, 442, 337

Popham, R., Woosley, S. E., \& Fryer, C. 1999, ApJ, 518, 356

Portegies-Zwart, S. F., Lee, C. H., \& Lee, H. K. 1999, ApJ, 520, 666

Riffert, H., \& Herold, H. 1995, ApJ, 450, 508

Romero, G. E., Torres, D. F., Andruchow, I., \& Anchordoqui, L. A. 1999, MNRAS, 308, 799

Setiawan, S., Ruffert, M., \& Janka, H.-Th. 2004, MNRAS, 352, 753

Shakura, N. I., \& Sunyaev, R. A. 1973, A\&A, 24, 337

Shapiro, P., \& Teukolsky, S. 1983, Black Holes, White Dwarfs, and Neutron Stars (New York: Wiley)

Wilkins, D. C. 1972, Phys. Rev. D, 5, 814

Woosley, S. E. 1993, ApJ, 405, 273 